\title{
Barriers and enablers to physical activity in patients during hospital stay: a scoping review
}

Sven Jacobus Gertruda Geelen ${ }^{1 *+} \mathbb{D}$, Hanneke Corine van Dijk - Huisman ${ }^{2,3^{*}}{ }^{\text {, }}$, Robert Adriaan de Bie ${ }^{3,4}$, Cindy Veenhof ${ }^{5,6}$, Raoul Engelbert ${ }^{1,7}$, Marike van der Schaaf ${ }^{1,7 \dagger}$ and Antoine François Lenssen ${ }^{2,3+}$

\begin{abstract}
Background: Low levels of physical activity are common during the hospital stay and have been associated with negative health outcomes. Understanding barriers and enablers to physical activity during a hospital stay can improve the development and implementation of tailored interventions aimed at improving physical activity. Previous studies have identified many barriers and enablers, but a comprehensive overview is lacking. This study aimed to identify and categorize all published patient- and healthcare professional-reported barriers and enablers to physical activity during a hospital stay for acute care, using the Theoretical Domains Framework (TDF).
\end{abstract}

Methods: We conducted a scoping review of Dutch and English articles using MEDLINE, CINAHL Plus, EMBASE, PsycINFO, and Cochrane Library (inception to September 2020), which included quantitative, qualitative, and mixedmethods studies reporting barriers and enablers to physical activity during a hospital stay for acute care, as perceived by patients or healthcare professionals. Two reviewers systematically extracted, coded, and categorized all barriers and enablers into TDF domains.

Results: Fifty-six articles were included in this review (32 qualitative, 7 quantitative, and 17 mixed-methods). In total, 264 barriers and 228 enablers were reported by patients, and 415 barriers and 409 enablers by healthcare professionals. Patient-reported barriers were most frequently assigned to the TDF domains Environmental Context \& Resources ( $E C R, n=148)$, Social Influences $(n=32)$, and Beliefs about Consequences $(n=25)$, while most enablers were assigned to ECR $(n=67)$, Social Influences $(n=54)$, and Goals $(n=32)$. Barriers reported by healthcare professionals were most frequently assigned to ECR $(n=210)$, Memory, Attention and Decision Process $(n=45)$, and Social/Professional Role \& Identity $(n=31)$, while most healthcare professional-reported enablers were assigned to the TDF domains $E C R(n=143)$, Social Influences $(n=76)$, and Behavioural Regulation $(n=54)$.

\footnotetext{
*Correspondence: s.j.geelen@amsterdamumc.nl; hanneke.huisman@mumc.

$\mathrm{nl}$

†'Sven Jacobus Gertruda Geelen and Hanneke Corine van Dijk - Huisman

shared first authorship.

${ }^{\dagger}$ Marike van der Schaaf and Antoine François Lenssen shared last

authorship.

${ }^{1}$ Department of Rehabilitation Medicine, Amsterdam Movement

Sciences, Amsterdam UMC, University of Amsterdam, Meibergdreef 9,

Amsterdam 1105AZ, The Netherlands

${ }^{2}$ Department of Physical Therapy, Maastricht University Medical Centre, P.

Debyelaan 25, Maastricht 6229HX, The Netherlands

Full list of author information is available at the end of the article
}

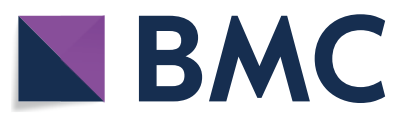

(c) The Author(s) 2021. Open Access This article is licensed under a Creative Commons Attribution 4.0 International License, which permits use, sharing, adaptation, distribution and reproduction in any medium or format, as long as you give appropriate credit to the original author(s) and the source, provide a link to the Creative Commons licence, and indicate if changes were made. The images or other third party material in this article are included in the article's Creative Commons licence, unless indicated otherwise in a credit line to the material. If material is not included in the article's Creative Commons licence and your intended use is not permitted by statutory regulation or exceeds the permitted use, you will need to obtain permission directly from the copyright holder. To view a copy of this licence, visit http://creativecommons.org/licenses/by/4.0/. The Creative Commons Public Domain Dedication waiver (http://creativeco mmons.org/publicdomain/zero/1.0/) applies to the data made available in this article, unless otherwise stated in a credit line to the data. 
Conclusions: Our scoping review presents a comprehensive overview of all barriers and enablers to physical activity during a hospital stay and highlights the prominent role of the TDF domains ECR and Social Influences in hospitalized patients' physical activity behavior. This TDF-based overview provides a theoretical foundation to guide clinicians and researchers in future intervention development and implementation.

Scoping review registration: No protocol was registered for this review.

Keywords: Physical activity, Mobility, Hospital, Barrier, Enabler, Theoretical domains framework

\section{Contributions to the literature}

- Physical inactivity during the hospital stay is a frequent problem, but an overview of patient- and healthcare professional-reported barriers and enablers to physical activity was lacking.

- The majority of barriers and enablers were categorized under the TDF-domains Environmental Context and Resources and Social Influences, highlighting the need for interventions that target the physical environment, hospital care processes, organizational characteristics, resources, patient-related factors, and social influences.

- Our comprehensive theory-informed overview of all published barriers and enablers to physical activity during a hospital stay can assist clinicians and researchers in developing and implementing tailored interventions in local clinical practice.

\section{Background}

Hospitalized patients spend between 87 and $100 \%$ of their time lying in bed or sitting, irrespective of the reason for admission [1]. Low levels of physical activity have been associated with negative health outcomes like functional decline [2, 3], increased length of stay [4], increased risk of institutionalization [5, 6], and mortality $[2,3,7,8]$. Previous research has shown that these negative health outcomes of inactivity can be counteracted by increasing physical activity levels [9-13]. Thus, interventions aimed at increasing the physical activity levels of hospitalized patients are of great importance [14].

Many different barriers and enablers influence patients' physical activity behavior [14-20]. While barriers reduce or negatively affect a patient's physical activity behavior $[15,18,21]$, enablers enhance or positively affect this behavior [14, 16, 19, 20]. Brown et al. have investigated barriers to physical activity in older adults admitted to a medical ward [15]. They identified having symptoms (e.g., weakness, pain, fatigue), being concerned about falls, and a lack of staff to assist with out-of-bed physical activity as frequently reported barriers. So et al. also described not being provided with adequate walking aids and being attached to an intravenous line as barriers [14]. Moreover, they identified many enablers, such as being encouraged to exercise, preventing the negative effects of prolonged bed rest, and promoting functional recovery.

Over the past two decades, the number of studies identifying barriers or enablers to physical activity during a hospital stay for acute care has grown significantly [1421]. In these studies, barriers and enablers were identified in a wide variety of patient populations and clinical settings [14-21]. Furthermore, they were explored from the perspective of patients $[14,20]$, healthcare professionals (HCPs) [16-18, 21], or both [15, 19]. To our knowledge, no comprehensive overview of barriers and enablers to physical activity during a hospital stay for acute care has been published. Such a comprehensive overview would provide clinicians and researchers with a better understanding of these barriers and enablers. This might improve the development of future interventions or implementation of existing interventions in different health care settings.

To be able to use such an overview in future intervention development or translation, it is essential to adopt a theoretical framework that links barriers and enablers to intervention strategies. A theoretical framework can help to guide interventions targeting modifiable factors for physical activity during the hospital stay for acute care $[22,23]$. Moreover, using a theoretical framework to identify barriers and enablers to behavioral change has been demonstrated to be more successful in changing behavior than using a non-theory-driven approach [24, 25].

One such integrative theoretical framework is the Theoretical Domains Framework (TDF) [25]. The TDF facilitates a systematic and theoretically based approach to behavior change. The validated TDF contains 14 domains, comprising 84 theoretical constructs from 33 theories of behavior and behavior change. Barriers and enablers can be categorized in the following domains: Knowledge, Skills, Social/Professional Role and Identity (SPRI), Beliefs About Capabilities, Optimism, Beliefs about Consequences, Reinforcement; Intentions, Goals, Memory, Attention and Decision Processes (MADP), Environmental Context and Resources (ECR), Social Influences, Emotion, and Behavioural Regulation. The 
TDF has been extensively used as a guide to identify and categorize modifiable factors that influence behavior [25]. The objective of this review was to identify and categorize patient- and HCP-reported barriers and enablers to physical activity during a hospital stay for acute care, using the TDF.

\section{Methods \\ Study design}

A scoping review was performed to explore the nature and quantity of published literature on barriers and enablers to physical activity during a hospital stay for acute care, as perceived by hospitalized patients and their HCPs. We used the scoping review methodology suggested by Arksey and O'Malley [26] and developed further by Levac, Colquhoun, and O'Brien [27, 28]. The Joanna Briggs Institute (JBI) guidance document for the conduct of scoping reviews and the Preferred Reporting Items for Systematic Reviews and Meta-Analyses Extension for Scoping Review (PRISMA-ScR) were used to inform the methodology (Additional file 1) [29, 30]. The TDF was used to categorize the barriers and enablers extracted from the included studies [25], as described in further detail in "Collating, summarizing, and reporting the results". No protocol was registered for this review.

\section{Search strategy and study selection}

A comprehensive search strategy was developed in collaboration with an experienced research librarian $(\mathrm{FvE})$ of the University of Amsterdam (Additional file 2). An electronic database search of MEDLINE (through Pubmed), CINAHL Plus, Cochrane, EMBASE, PsycINFO, and the Cochrane library was performed, from the inception of the electronic databases to September 23, 2020.

All electronic database searches were combined and de-duplicated in Endnote version X9.1 (Clarivate Analytics, Philadelphia, Pennsylvania, USA) [31]. Two reviewers (SJGG and $\mathrm{HCvDH}$ ) independently screened all titles and abstracts to determine eligibility, based on the following inclusion and exclusion criteria. Studies were considered eligible if they reported barriers or enablers to physical activity during a hospital stay as perceived by patients or HCPs. Patients had to be hospitalized in an acute care setting and HCPs had to be involved in clinical care (e.g., physicians, nurses, nursing assistants, occupational therapists, and physiotherapists). Barriers were defined as any factor reducing or negatively affecting a patient's engagement in physical activity. Enablers were defined as any factor enhancing or positively affecting a patient's engagement in physical activity. Barriers and enablers had to be self-reported. Studies reporting factors associated or correlated to physical activity during the hospital stay for acute care that was not self-reported were not included in this study [32]. Published full-text articles using quantitative, qualitative, or mixed-method study designs were considered, as was gray literature (i.e., academic papers, theses, and dissertations). Only studies written in English or Dutch were included. Studies reporting solely on children ( $<18$ years), short-stay admissions $(<24 \mathrm{~h})$, the Intensive Care Unit, or psychiatric ward were excluded because of the differences in care and context (e.g., in terms of organization of care, length of hospital stay, patient characteristics, and care provided). Protocols and reviews were excluded as they lack empirical data. Case studies were also excluded as they often describe extreme cases that do not represent the general population of hospitalized patients. Lastly, conference abstracts were excluded.

To ensure that at least $80 \%$ agreement was reached between the reviewers in determining eligibility based on study titles and abstracts, a pilot was performed using $5 \%$ of the references. The pilot resulted in minor revisions of the inclusion and exclusion criteria, to enhance the clarity of the criterion descriptors. Full-text articles were obtained when studies fulfilled the criteria or when additional information was needed to determine eligibility. Subsequently, full-text articles were independently screened by both reviewers to determine eligibility. To ensure that at least $80 \%$ agreement was reached between reviewers in determining eligibility based on full texts, a pilot was first performed using $10 \%$ of the references.

To reduce the risk of missing relevant studies, reference lists of included studies and the reviewers' own literature databases were screened for additional studies. Any disagreements during the study selection process were resolved by discussion, mediated by a senior researcher (AFL). The web application of Rayyan QCRI (Qatar Computing Research Institute, Hamad Bin Khalifa University) was used to facilitate the study selection process [33]. A PRISMA-ScR flowchart was created to track the screening and inclusion process of this review [30,33].

\section{Data extraction}

Both reviewers (SJGG and $\mathrm{HCvDH}$ ) independently extracted data using a custom-built data extraction form. Characteristics of included studies (author(s), year of publication, type of study, study aim, method, population, setting, and study sample) were extracted according to the JBI Guidance document for the conduct of scoping reviews [29]. Barriers and enablers identified in the results sections of the included studies were extracted using an iterative data extraction process. Barriers and enablers reported by patients and HCPs were extracted separately. Different extraction methods were used for qualitative and quantitative studies [34]. From qualitative studies, all barriers and enablers reported by 
patients or HCPs were extracted. For quantitative studies, the approach described by Weatherson [35] was used, meaning that barriers and enablers were extracted if $\geq 50 \%$ of participants agreed that the factor influenced patients' physical activity behavior. For example, in a survey with dichotomous answering options (agree/ disagree), the factor "discussing physical activity during physician rounds increases patients' physical activity levels" was not extracted as an enabler if $42 \%$ of the HCPs agreed. Some questionnaire measures contained an intermediate category, such as 5-point Likert-scale questions with answering options: $1=$ strongly agree, $2=$ somewhat agree, $3=$ neither agree nor disagree, $4=$ somewhat disagree, and $5=$ strongly disagree. Barriers or enablers were only extracted if at least $50 \%$ of participants somewhat agreed or strongly agreed that they perceived it as a barrier or enabler [35]. For example, if $60 \%$ of the HCPs agreed (18\% somewhat agreed and $42 \%$ strongly agreed) that "discussing physical activity during physician rounds increases patients' physical activity levels" was an enabler, this factor was extracted as enabler [35]. If a quantitative study included openended questions, the responses were extracted as in qualitative studies.

To ensure the reliability of the data extraction process, the reviewers first extracted data from five randomly selected articles [14-16, 19, 36] and discussed their findings to resolve disagreements and improve the preliminary data extraction table. This process was then repeated with five other articles [17, 21, 37-39], after which both researchers agreed on the data extraction and no further changes to the data extraction table were required. Finally, each reviewer independently extracted half of the remaining articles and then critically reviewed the extraction of the other half performed by the other reviewer. Disagreements were resolved by discussion and rereading source material, and two senior researchers were consulted in case of discrepancies (AFL and MvdS).

\section{Collating, summarizing, and reporting the results}

Both reviewers (SJGG and $\mathrm{HCvDH}$ ) independently coded the extracted barriers and enablers and categorized them into the 14 TDF domains [25, 40,41]. The theoretical definitions and component constructs of the domains as presented in Additional file 3 were used to guide the coding process. Barriers and enablers were coded separately for patients and HCPs and were coded to more than one domain if the content suited multiple domains. To increase inter-coder reliability, the two reviewers (SJGG and $\mathrm{HCvDH}$ ) met to discuss coding discrepancies and to iteratively modify the coding structure after every ten articles. Discrepancies were solved by discussion and rereading the articles. If necessary, a senior researcher (MvdS) was consulted to discuss and resolve discrepancies. This process was repeated until a final TDF categorization had been obtained. Two senior researchers (AFL and MvdS) supervised the categorization process. The entire authorship team reviewed the final categorization. MAXQDA Analytics Plus 2020 (VERBI Software, 2018, Berlin, Germany) was used to facilitate data coding and the categorization process. The numbers of different barriers and enablers assigned per TDF domain as well as the number of articles reporting on barriers and enablers per TDF domain were presented separately for patients and HCPs. Finally, a descriptive summary of the reported barriers and enablers was composed for patients and HCPs.

\section{Results}

The search retrieved 6716 studies, of which 2382 were excluded as duplicates. An additional three studies [4244] were retrieved by hand-searching the researchers' own literature database (i.e., two studies which did not explicitly mention "barrier," "enabler," or "hospital" in the title and abstract, and one which was a Masters thesis). A total of 4334 studies were screened based on titles and abstracts. Of the 143 articles that were assessed as full texts, 45 were identified for inclusion [11, 14-16, 18-21, 36-39, 42-74]. An additional 11 studies were included after hand-searching the reference lists of included studies $[17,75-84]$, resulting in a total of 56 included studies [11, 14-21, 36-39, 42-84]. The PRISMA-ScR flowchart (Fig. 1) shows the screening and inclusion process.

\section{Description of included studies}

Additional file 4 presents an overview of the included studies. Of the 56 studies, 32 used a qualitative study design [14-20, 38, 42, 44-46, 49-54, 57, 61, 67, 68, 70, $72-76,78-80,83]$, seven a quantitative study design [21, $37,39,63,66,69,82]$, and 17 a mixed-methods study design $[11,36,43,47,48,55,56,58-60,62,64,65,71,77$, $81,84]$. Nineteen studies reported barriers and enablers as perceived by patients $[14,20,36,37,39,48,51,61,63-$ $65,67,70,73,78,79,81,83], 23$ reported those perceived by HCPs $[16-18,21,38,42,43,47,50,52-55,57,59,60$, $68,69,74-76,80,82]$, and 14 reported those perceived by patients and HCPs $[11,15,19,44-46,49,56,58,62$, $66,71,72,77,84]$. Sample sizes varied between $n=6$ and $n=345$ patients and between $n=5$ and $n=261$ HCPs. Two studies did not specify the sample size $[11,77]$, and one study only specified the number of included sites [47]. Further descriptions of the populations and settings included are provided in Additional file 4. The included studies were published between 2003 and 2020, and only 


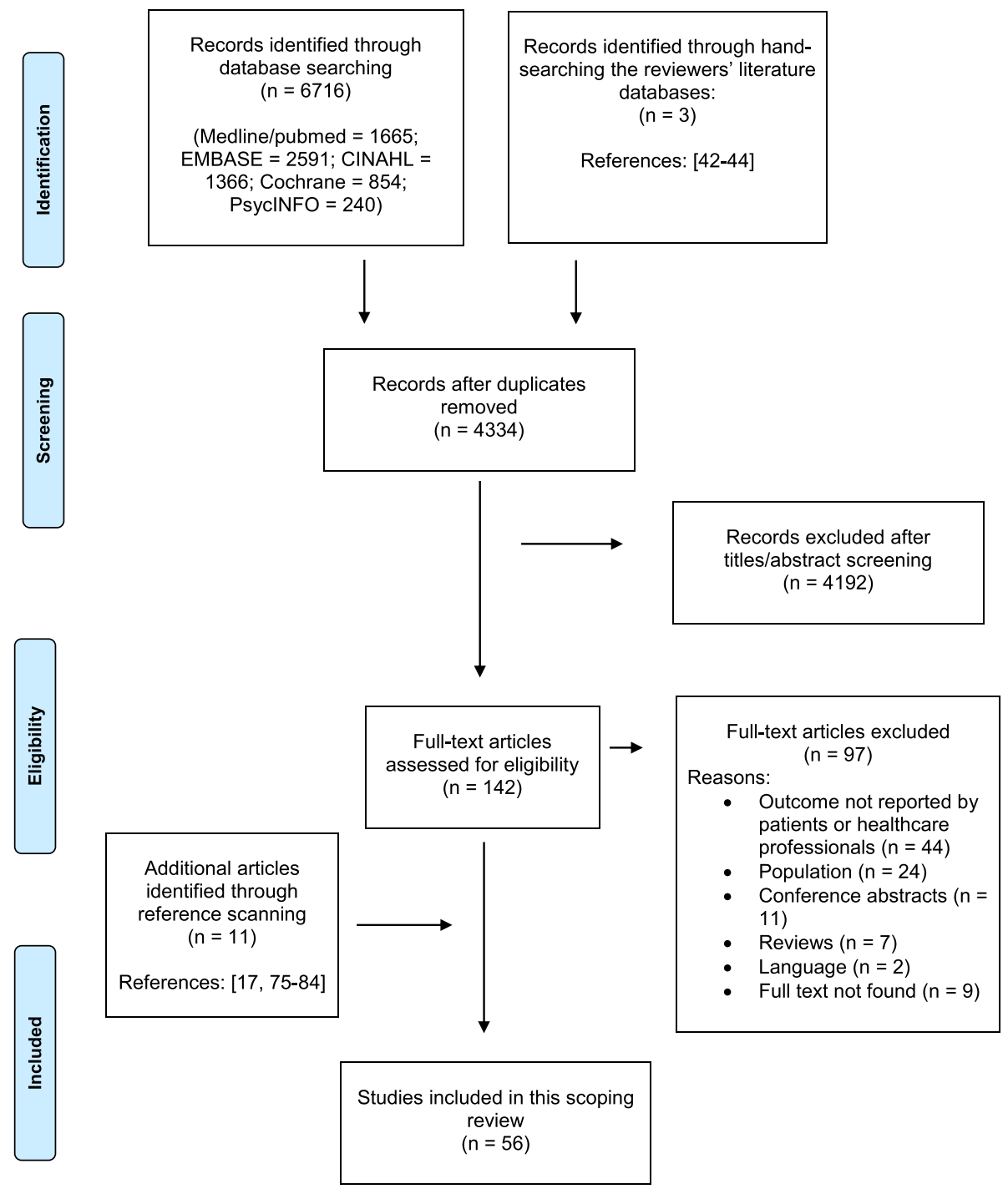

Fig. 1 PRISMA-SCR flowchart

seven studies were published before 2010 [15, 52, 56, 75, $77,78,82]$.

\section{Identification of patient- and HCP-reported barriers and enablers to physical activity during a hospital stay for acute care}

The results of the data extraction process are presented in Additional file 5. After coding and discussing all extracted fragments containing barriers and enablers, SJGG and $\mathrm{HCVDH}$ reached a consensus on 1316 barriers and enablers. Two hundred sixty-four (20.2\%) patient-reported barriers and 415 (31.7\%) HCP-reported barriers were coded. Two hundred twenty-eight (17.3\%) patient-reported enablers and 409 (31.2\%) HCP-reported enablers were coded.

\section{Categorizing patient- and HCP-reported barriers using the TDF}

Patient- and HCP-reported barriers were assigned to 13 of the 14 TDF domains. An overview of the TDF coding of all barriers is provided in Additional file 6 and summarized in Fig. 2. Patient-reported barriers were assigned most frequently to the TDF domains ECR $(n=148$, $56.1 \%)$, Social Influences $(n=32,12.1 \%)$, and Beliefs about Consequences $(n=25,9.5 \%)$. Of the other 11 domains, the largest numbers of barriers were assigned to the domains Emotion $(n=16,6.1 \%)$ and SPRI $(n=$ $10,3.8 \%)$. HCP-reported barriers were assigned most frequently to the TDF domains $E C R(n=210,50.6 \%)$, $\operatorname{MADP}(n=45,10.8 \%)$, and SPRI $(n=31,7.5 \%)$. Of the other 11 domains, the largest numbers of barriers were 


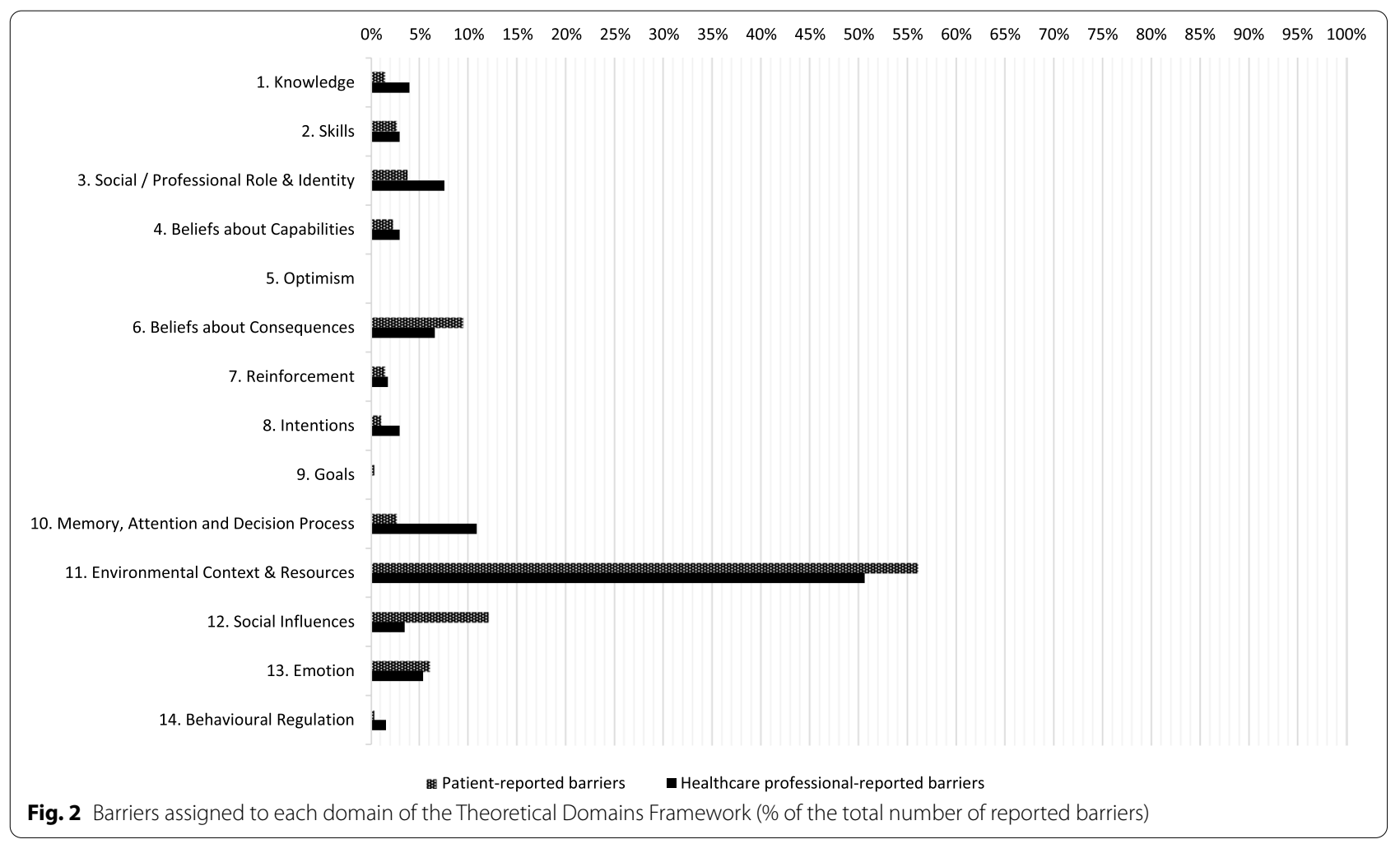

assigned to the domains Beliefs about Consequences $(n=27,6.5 \%)$ and Emotion $(n=22,5.3 \%)$. No patientand HCP-reported barriers were assigned to the domain Optimism.

The TDF domains to which barriers were most frequently assigned are highlighted below. The domain $E C R$ had the majority of both patient- and HCP-reported barriers assigned to it and covered four main topics: (1) patient-related factors (e.g., medical factors, age, language barriers), (2) care processes and organizational characteristics (e.g., prescribed immobility, communication, hospital culture, bed-centered care), (3) physical environment of the hospital (e.g., room, unit, hospital), and (4) resources (e.g., limited time, staffing, equipment) (Additional file 6). Patient-reported barriers assigned to the domain Social Influences included interpersonal processes between patients, visitors, and HCPs that negatively influence physical activity, such as lack of encouragement and assistance and providing more care than necessary. Patient-reported barriers assigned to the domain Beliefs about Consequences included the belief that physical activity results in negative consequences (e.g., injuries, falling, or missing meals and care), the belief that rest is needed for recovery, and the belief that patients may be inconveniencing busy HCPs. Most of the HCP-reported barriers assigned to the domain $M A D P$ related to prioritization. A high workload and safety considerations resulted in physical activity receiving a lower priority than medical treatment or rest. HCPreported barriers assigned to the domain SPRI included the passive and dependent attitude patients adopt during hospitalization (e.g., the idea that patients should remain in bed, personality, and character traits). In addition, HCPs mentioned the role they fulfill regarding physical activity (e.g., lack of role clarity in improving physical activity, attributing responsibility to others, and nurses lacking autonomy in deciding how and when to mobilize patients).

\section{Categorizing patient- and HCP-reported enablers using the TDF}

Patient- and HCP-reported enablers were assigned to 11 and 13 of the 14 TDF domains, respectively. An overview of the TDF-coding of all enablers is provided in Additional file 7 and summarized in Fig. 3. Patient-reported enablers were most frequently assigned to the TDF domains ECR ( $n=67,30.2 \%)$, Social Influences $(n=54$, $24.3 \%$ ), and Goals ( $n=32,14.4 \%)$. Of the remaining 11 domains, the largest numbers of enablers were assigned to the domains Knowledge $(n=24,10.5 \%)$ and Beliefs about Consequences $(n=17,7.7 \%)$. No patient-reported enablers were assigned to the domains Reinforcement, $M A D P$, and Emotion. HCP-reported enablers were most frequently assigned to the TDF domains $\operatorname{ECR}(n=143$, 


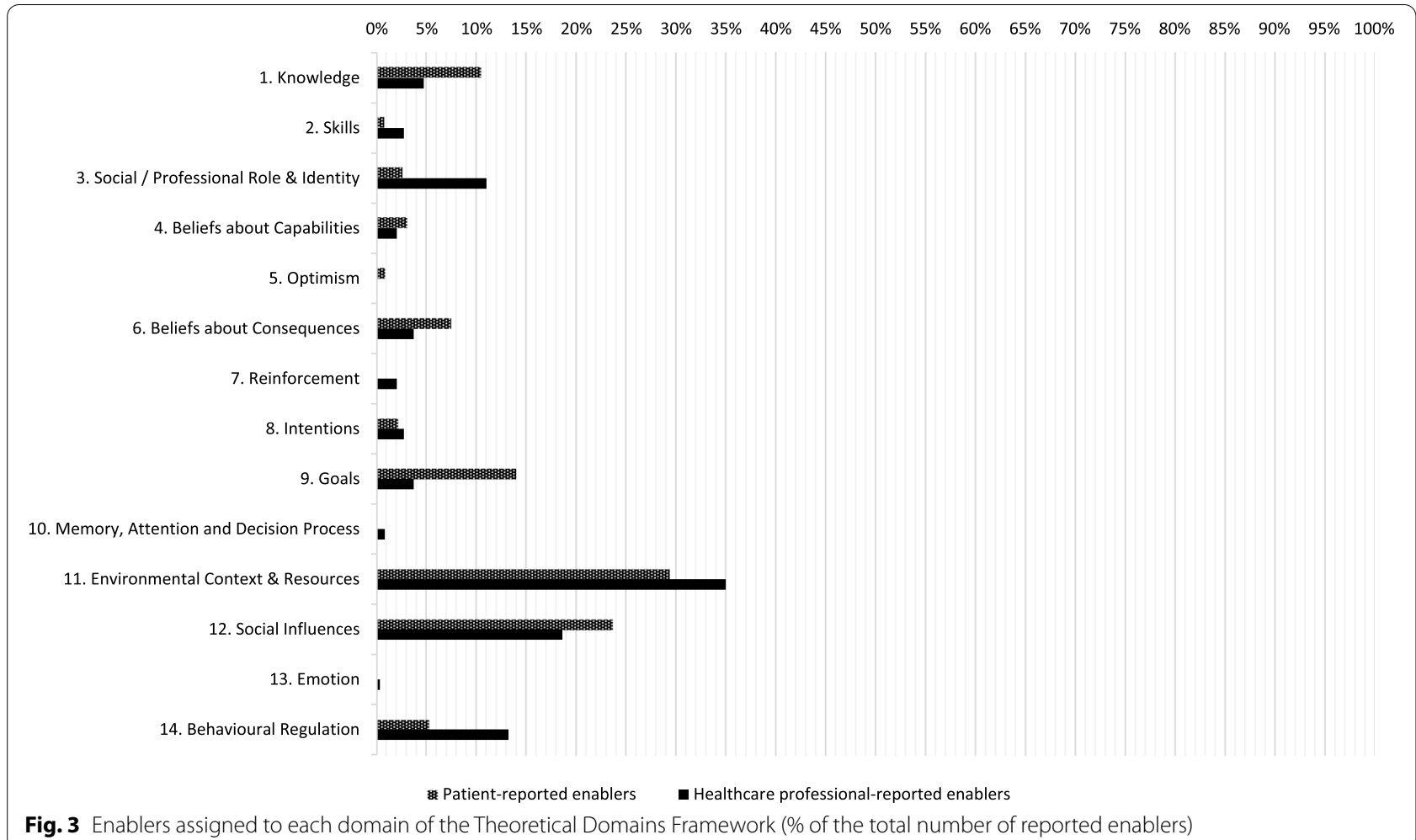

$35.0 \%)$, Social Influences $(n=76,18.6 \%)$, and Behavioral Regulation ( $n=54,13.2 \%)$. Of the remaining 11 domains, the largest numbers of enablers were assigned to the domains SPRI $(n=45,11 \%)$ and Knowledge $(n=$ $19,4.7 \%)$. No HCP-reported enablers were assigned to the domain Optimism.

In line with the categorization of the barriers, most patient- and HCP-reported enablers were assigned to the domain ECR and covered the same four main topics: (1) patient-related factors, (2) care processes and organizational characteristics, (3) physical environment of the hospital, and (4) resources (Additional file 7). Patientand HCP-reported enablers assigned to the domain Social Influences included interpersonal processes between patients and visitors or HCPs that positively influence physical activity, such as being encouraged and assisted. Patients also described that other patients motivated them to perform more physical activity, while HCPs described how leadership and multidisciplinary collaboration enabled them to improve patients' physical activity. Patient-reported enablers assigned to the domain Goals included the importance of having a goal (e.g., experiencing the positive effects of physical activity or preventing the negative effects of physical inactivity). This domain also included the importance of having autonomy and being involved in physical activity-related decision-making. HCP-reported enablers assigned to the domain Behavioural Regulation included strategies aimed at regulating behavior, such as providing education, appointing mobility champions, making performance and expectations visible, creating a habit, and using mobility documentation tools, reminders, daily schedules, exercise programs, and mobility audits.

\section{Discussion}

The aim of this study was to identify and categorize patient- and HCP-reported barriers and enablers to physical activity during a hospital stay for acute care, using the TDF. Our systematic search identified 679 barriers and 637 enablers, reported in 56 studies. The majority of barriers and enablers were assigned to the key domain Environmental Context and Resources (i.e., "patient-related factors," "care processes and organizational characteristics," "physical environment of the hospital," and "resources"). Other key TDF domains to which the largest numbers of barriers were assigned were Social Influences, Beliefs about Consequences, Memory, Attention and Decision Process, and Social/ Professional Role E Identity. Additionally, other key domains to which the largest numbers of enablers were assigned were Social Influences, Goals, and Behavioural Regulation. This is the first scoping review of patientand HCP-reported barriers and enablers relating to physical activity during the hospital stay for acute care 
using a TDF analysis. This review presents a comprehensive overview of these barriers and enablers from a theoretical perspective, which can help clinicians and researchers identify and target modifiable factors within future intervention development.

Our findings highlight the prominent role of the domain Environmental Context and Resources with respect to physical activity during the hospital stay for acute care. Upon hospital admission, patients are taken out of their own environment and enter a different, unfamiliar context, filled with many uncertainties. In addition to patients' illness and associated medical factors, the hospital environment exerts an inactivating influence on patients, resulting in a loss of autonomy and freedom $[15,44]$. Our findings indicate that "care processes and organizational characteristics," the "physical environment," "patient-related factors," and "resources" are the main topics of the domain Environmental Context and Resources that influence the physical activity behavior of hospitalized patients. Several studies have aimed to improve physical activity in hospitalized patients by targeting these main environmental factors [11, 12, 71, 85]. "Care processes and organizational characteristics" was targeted by incorporating physical activity in usual care $[12,85,86]$, creating policy to promote mobility [71], incorporating specific timeslots for physical activity in HCPs' schedules [71], improving communication [12], and providing patients with graded exercise programs [11]. "Physical environment" was targeted by providing interesting walking destinations [11], marked walking trails [71], distance markers in the hallway [71], ward maps and signs [11], and by making mobilization goals visible $[12,86]$. "Patient-related factors" were targeted by optimizing pain control [12], and "resources" by purchasing more walking aids [71], supporting physical activity with technology [86, 87], and supplying activity diaries and exercise booklets $[11,85]$.

Our results also highlight the role of the domain Social Influences, identified as the second most prominent TDF domain. The absence of encouragement and assistance by others (i.e., nurses, physical therapists, physicians, visitors, volunteers, or other patients) was identified as an important barrier by patients, whereas their presence as an important enabler. This was substantiated by HCPs, who also added multidisciplinary teamwork, leadership support, the presence of physical therapists, and involving visitors as important enablers of physical activity. Several studies have aimed to improve physical activity by targeting the domain Social Influences, by providing systematic encouragement and assistance from HCPs [11, 71, 85, 86], involving volunteers or family members in basic mobility activities [11, 86], and encouraging independence in activities of daily living [11].
Moreover, the domains Beliefs about Consequences, Memory, Attention and Decision Process, and Social/Professional Role \& Identity also contained many barriers. Several studies have targeted these domains to improve patients' physical activity levels, such as providing education to counter the belief that physical activity will result in injuries $[86,88]$, using shift huddles to address prioritizing physical activity [89], or mapping the therapy consultation process within a multidisciplinary team to create role clarity and avoid unnecessary treatments [90]. Likewise, the domains Goals and Behavioural Regulation contained many enablers. Examples of interventions that specifically focus on goal setting and behavioral regulation are the Johns Hopkins Highest Level of Mobility tool [12], the WALK-FOR 900 steps per day behavioral target [91], and Hospital Fit monitor [87]. All these interventions enable monitoring physical activity levels and setting physical activity goals in daily clinical care.

Our findings indicated that there were several TDF domains (e.g., Skills, Optimism, Reinforcement) to which few or no barriers and enablers were assigned. The many factors assigned to the TDF domains Environmental Context and Resources and Social Influences, and the few factors assigned to the domains Skills, Optimism, and Reinforcement are in agreement with the results of similar research performed in other populations, such as physical activity at school [35], work [92], or in primary care [93]. Although this highlights the prominent role of the domains Environmental Context and Resources and Social Influences on physical activity behavior, it does not indicate whether the domains Skills, Optimism, and Reinforcement do not contain relevant barriers and enablers to physical activity, or whether they were under-identified.

Lastly, although many patient-reported barriers and enablers were also reported by HCPs, our results demonstrated that HCPs perceived a greater number of barriers and enablers than patients. This could be explained by the different perspectives of patients and HCPs on physical activity during the hospital stay. Patients are hospitalized for a relatively short period, with their main focus being their illness and getting better. They experience how it feels to be a patient and how this influences their physical activity behavior. On the other hand, HCPs perceive barriers and enablers from a much broader perspective. Firstly, they report barriers and enablers from their own as well as their patients' perspectives. Secondly, they provide care to many patients with different pathologies, ages, and backgrounds. Thirdly, they perceive barriers and enablers related to providing care, different care processes, and organizational characteristics. These differences in perspectives between patients and HCPs emphasize that both must be taken into account to gain 
a comprehensive understanding of the barriers and enablers to physical activity during a hospital stay.

\section{Strengths and limitations}

This is the first scoping review on patient- and HCPreported barriers and enablers relating to physical activity during the hospital stay for acute care using a TDF analysis. A strength of this study is that it was designed and conducted according to the systematic scoping review methodology and that it followed the PRISMAScR statement recommendations [26-30]. Secondly, almost all aspects of data collection, data extraction, and data analysis were carried out independently by two researchers, with a third party available in case of disagreements. Thirdly, given the extensive, thorough search strategy in multiple databases, along with the inclusion of quantitative, qualitative as well as mixed-methods study designs, we were able to present a complete overview of all barriers and enablers reported in the current literature. Fourthly, an additional strength of this study is the use of the TDF as a theoretical framework to categorize barriers and enablers. The use of the TDF ensured that the reviewers assessed barriers and enablers from a broad perspective, thereby also exploring underexposed domains.

We also recognize some limitations. While the use of the TDF facilitates reviewers in exploring barriers and enablers from a broad perspective, it does not provide an explanation as to how barriers and enablers are connected and influence one another. Another limitation of this study is that barriers and enablers are presented based on the number of articles in which they have been reported. As the frequency of reporting is primarily a function of the methods used to present the data, this alone should not be used as a proxy of importance. In other words, a barrier that has only been reported once may be just as relevant as one that has been reported many times. Furthermore, a secondary analysis of differences in perceived barriers and enablers among patient subgroups or among professions could not be performed due to the lack of detailed reporting in the included studies. Lastly, as this was a scoping review, no quality appraisal of included articles was performed [30].

\section{Clinical implications and recommendations for future research}

Our findings provide a comprehensive overview of barriers and enablers to physical activity during a hospital stay for acute care. The large number of barriers and enablers we found, distributed across many TDF domains, highlight the complexity of physical activity behavior during the hospital stay and the need for tailored interventions. A context-based assessment should be performed to determine which barriers and enablers can be targeted in a specific clinical setting. Our comprehensive overview will enable clinicians and researchers to perform this context-based assessment from a broad perspective and support them in establishing a behavioral diagnosis of what needs to change in a specific context in order to improve physical activity behavior during the hospital stay.

Subsequently, clinicians and researchers will be able to link relevant barriers and enablers to specific intervention strategies and behavior change techniques (BCTs) [25, $41,94]$. An example of a framework that could be used to assist clinicians and researchers in selecting appropriate BCTs is the Behaviour Change Wheel (BCW) [22]. Our TDF-based overview provides the initial step in developing and implementing theory-informed behavior change interventions aimed at improving physical activity during the hospital stay [41].

Given the large number of factors influencing the physical activity behavior of hospitalized patients, we recommend that clinicians and researchers develop and implement interventions targeted at multiple barriers and enablers. Previous research suggests that developing and implementing such tailored multimodal interventions may be more effective than unimodal interventions [95]. Moreover, given a large number of barriers and enablers assigned to the Environmental Context and Resources and Social Influences context in our review, we suggest that clinicians and researchers should always consider incorporating intervention strategies targeting these TDF domains in their multimodal interventions.

Future research should focus on exploring relationships between barriers and enablers both within and between TDF domains. Revealing these relationships may facilitate the assessment of barriers and enablers in specific clinical settings and may increase the effectivity of future tailored multimodal interventions. Future research is also needed to explore the differences in perspectives perceived by different patient subgroups (e.g., age, sex, pathologies). Similarly, more research is needed to investigate differences in perceived barriers and enablers among professions and how these differences relate to their role in improving physical activity during the hospital stay. Additionally, further research is needed to develop and validate a TDF-based questionnaire that could facilitate the context-based assessment of barriers and enablers across all TDF domains. Further research is needed to retrospectively identify which barriers and enablers to physical activity during the a hospital stay have been targeted in previously described intervention studies [94], so clinicians may be better able to implement these interventions in other contexts. Finally, there is a need for research assessing the effectiveness of tailored multimodal interventions that target context-based 
barriers and enablers to physical activity in hospitalized patients.

\section{Conclusions}

This article presents a comprehensive overview of 1316 patient- and HCP-reported barriers and enablers to physical activity during a hospital stay for acute care. A large number of barriers and enablers found highlight the complexity of physical activity behavior during the hospital stay. Our overview can assist clinicians and researchers in performing a context-based assessment to determine which barriers and enablers to target in future interventions. Given the large number of factors influencing the physical activity behavior of hospitalized patients, we recommend developing and implementing multimodal interventions. This scoping review also highlights the large role of environmental and social factors on physical activity behavior during the hospital stay and suggests that intervention strategies targeting these domains should be incorporated. Future research should focus on exploring the relationships between barriers and enablers both within and between different TDF domains. Revealing these relationships may facilitate the assessment of barriers and enablers in specific clinical settings and may increase the effectivity of future tailored multimodal interventions. Furthermore, future research is also needed to explore the differences in perspectives perceived among different patient subgroups or different professions. Lastly, a validated TDF-based questionnaire is needed to facilitate future context-based assessments of barriers and enablers, and further research should investigate the effectiveness of tailored multimodal interventions.

\section{Abbreviations}

HCPs: Healthcare professionals; JBI: Joanna Briggs Institute; PRISMA-ScR: Preferred Reporting Items for Systematic Reviews and Meta-Analyses Extension for Scoping Reviews; TDF: Theoretical Domains Framework; UK: United Kingdom; USA: United States of America.

\section{Supplementary Information}

The online version contains supplementary material available at https://doi. org/10.1186/s13643-021-01843-x.

Additional file 1. PRISMA-ScR checklist. Completed PRISMA-ScR checklist for this scoping review.

Additional file 2. Search terms. Presentation of the used search terms with databases.

Additional file 3. The Theoretical Domains Framework with definitions and component constructs. Description of the definitions and component constructs of each TDF domain.

Additional file 4. Characteristics of included studies. Table presenting the characteristics of included studies.

Additional file 5. Data extraction. Excel file presenting all extracted data from included studies.
Additional file 6. Barriers to physical activity during hospital stay for acute care as reported by patients and healthcare professionals. Table presenting the barriers to physical activity.

Additional file 7. Enablers to physical activity during a hospital stay for acute care as reported by patients and healthcare professionals. Table presenting the enablers to physical activity.

\section{Acknowledgements}

We would like to thank the research librarian Faridi van Etten for her assistance with the search strategies and the de-duplication of all titles and abstracts. We would also like to thank Jan Klerkx who provided language editing services.

\section{Authors' contributions}

SJGG and HCVDH declare equal first authorship, as both contributed equally to conducting the scoping review and writing the manuscript. RAdB, CV, and RE have participated in conceiving the scoping review, commenting on the review process, interpreting the results, and critically reviewing earlier drafts. MvdS and AFL participated equally in designing the scoping review, supervising, commenting on the review process, interpreting the results, and critically reviewing earlier drafts; they therefore declare to share the senior authorship. The authors have read and approved the final manuscript.

\section{Funding}

No sources of funding were provided for this study.

\section{Availability of data and materials}

The datasets used and/or analyzed during the current study are available from the corresponding author on reasonable request.

\section{Declarations}

Ethics approval and consent to participate Not applicable.

Consent for publication

Not applicable.

Competing interests

The authors declare that they have no competing interests.

\section{Author details}

${ }^{1}$ Department of Rehabilitation Medicine, Amsterdam Movement Sciences, Amsterdam UMC, University of Amsterdam, Meibergdreef 9, Amsterdam 1105AZ, The Netherlands. ${ }^{2}$ Department of Physical Therapy, Maastricht University Medical Centre, P. Debyelaan 25, Maastricht 6229HX, The Netherlands. ${ }^{3}$ CAPHRI School for Public Health and Primary Care, Maastricht University, Maastricht, the Netherlands. ${ }^{4}$ Department of Epidemiology, Maastricht University, Maastricht, the Netherlands. ${ }^{5}$ Physical Therapy Research, Department of Rehabilitation, Physical Therapy Sciences \& Sports, University Medical Centre Utrecht, Utrecht University, Utrecht, The Netherlands. ${ }^{6}$ Expertise Centre Healthy Urban Living, Research Group Innovation of Human Movement Care, University of Applied Sciences Utrecht, Utrecht, The Netherlands. ${ }^{7}$ Centre of Expertise Urban Vitality, Faculty of Health, Amsterdam University of Applied Sciences, Amsterdam, the Netherlands.

Received: 4 February 2021 Accepted: 21 October 2021

Published online: 04 November 2021

\section{References}

1. Fazio S, Stocking J, Kuhn B, Doroy A, Blackmon E, Young HM, et al. How much do hospitalized adults move? A systematic review and meta-analysis. Appl Nurs Res. 2019;51:151189.

2. Buurman BM, Hoogerduijn JG, de Haan RJ, Abu-Hanna A, Lagaay AM, Verhaar $\mathrm{HJ}$, et al. Geriatric conditions in acutely hospitalized older patients: prevalence and one-year survival and functional decline. PLoS One. 2011;6(11):e26951. 
3. Boyd CM, Landefeld CS, Counsell SR, Palmer RM, Fortinsky RH, Kresevic D, et al. Recovery of activities of daily living in older adults after hospitalization for acute medical illness. J Am Geriatr Soc. 2008:56(12):2171-9.

4. Brown CJ, Friedkin RJ, Inouye SK. Prevalence and outcomes of low mobility in hospitalized older patients. J Am Geriatr Soc. 2004;52(8):1263-70.

5. Mahoney JE, Sager MA, Jalaluddin M. New walking dependence associated with hospitalization for acute medical illness: incidence and significance. J Gerontol A Biol Sci Med Sci. 1998;53(4):M307-12.

6. Brown CJ, Redden DT, Flood KL, Allman RM. The underrecognized epidemic of low mobility during hospitalization of older adults. J Am Geriatr Soc. 2009;57:1660-5.

7. Covinsky KE, Palmer RM, Fortinsky RH, Counsell SR, Stewart AL, Kresevic $D$, et al. Loss of independence in activities of daily living in older adults hospitalized with medical illnesses: increased vulnerability with age. J Am Geriatr Soc. 2003;51:451-8.

8. Ostir GV, Berges IM, Kuo YF, Goodwin JS, Fisher SR, Guralnik JM. Mobility activity and its value as a prognostic indicator of survival in hospitalized older adults. J Am Geriatr Soc. 2013;61(4):551-7.

9. Agmon M, Zisberg A, Gil E, Rand D, Gur-Yaish N, Azriel M. Association between 900 steps a day and functional decline in older hospitalized patients. JAMA Intern Med. 2017;177(2):272-4.

10. Brown CJ, Foley KT, Lowman JD Jr, MacLennan PA, Razjouyan J, Najafi B, et al. Comparison of posthospitalization function and community mobility in hospital mobility program and usual care patients: a randomized clinical trial. JAMA Intern Med. 2016;176(7):921-7.

11. Mudge AM, McRae P, Cruickshank M. Eat walk engage: an interdisciplinary collaborative model to improve care of hospitalized elders. Am J Med Qual. 2015;30(1):5-13.

12. Hoyer EH, Friedman M, Lavezza A, Wagner-Kosmakos K, Lewis-Cherry $R$, Skolnik JL, et al. Promoting mobility and reducing length of stay in hospitalized general medicine patients: a quality-improvement project. J Hosp Med. 2016;11(5):341-7.

13. Stolbrink M, McGowan L, Saman H, Nguyen T, Knightly R, Sharpe J, et al. The early mobility bundle: a simple enhancement of therapy which may reduce incidence of hospital-acquired pneumonia and length of hospital stay. J Hosp Infect. 2014;88:34-9.

14. So C, Pierluissi E. Attitudes and expectations regarding exercise in the hospital of hospitalized older adults: a qualitative study. J Am Geriatr Soc. 2012;60(4):713-8.

15. Brown CJ, Williams BR, Woodby LL, Davis LL, Allman RM. Barriers to mobility during hospitalization from the perspectives of older patients and their nurses and physicians. J Hosp Med. 2007;2:305-13.

16. Boltz M, Capezuti E, Shabbat N. Nursing staff perceptions of physical function in hospitalized older adults. Appl Nurs Res. 2011;24(4):215-22.

17. Doherty-King B, Bowers B. How nurses decide to ambulate hospitalized older adults: development of a conceptual model. Gerontologist. 2011:51:786-97.

18. Moore JE, Mascarenhas A, Marquez C, Almaawiy U, Chan WH, D'Souza J, et al. Mapping barriers and intervention activities to behaviour change theory for Mobilization of Vulnerable Elders in Ontario (MOVE ON), a multi-site implementation intervention in acute care hospitals. Implement Sci. 2014;9:160.

19. De Klein K, Valkenet K, Veenhof C. Perspectives of patients and healthcare professionals on physical activity of hospitalized patients. Physiother Theory Pract. 2021:37(2):307-14.

20. Boltz M, Capezuti E, Shabbat N, Hall K. Going home better not worse: older adults' views on physical function during hospitalization. Int J Nurs Pract. 2010;16(4):381-8.

21. Hoyer EH, Brotman DJ, Chan KS, Needham DM. Barriers to early mobility of hospitalized general medicine patients: survey development and results. Am J Phys Med Rehabil. 2015;94(4):304-12.

22. Michie S, Atkins L, West R. The behaviour change wheel: a guide to designing interventions. 1st ed. United Kingdom: Silverback Publishing; 2014. 647978-1-291-84605-8

23. Nilsen P. Making sense of implementation theories, models and frameworks. Implement Sci. 2015;10(1):53.

24. Gourlan M, Bernard P, Bortolon C, Romain AJ, Lareyre O, Carayol M, et al. Efficacy of theory-based interventions to promote physical activity. A meta-analysis of randomised controlled trials. Health Psychol Rev. 2016;10(1):50-66
25. Atkins L, Francis J, Islam R, O'Connor D, Patey A, Ivers N, et al. A guide to using the Theoretical Domains Framework of behaviour change to investigate implementation problems. Implement Sci. 2017;12(1):77.

26. Arksey H, O'Malley L. Scoping studies: towards a methodological framework. Int J Soc Res Methodol. 2005;8(1):19-32.

27. Colquhoun HL, Levac D, O'Brien KK, Straus S, Tricco AC, Perrier L, et al. Scoping reviews: time for clarity in definition, methods, and reporting. J Clin Epidemiol. 2014;67(12):1291-4.

28. Levac D, Colquhoun H, O'Brien KK. Scoping studies: advancing the methodology. Implement Sci. 2010;5:69.

29. Peters MDJ, Godfrey C, McInerney P, Baldini Soares C, Khalil H, Parker D. Chapter 11: Scoping reviews. In: Aromataris E, Munn Z, editors. Joanna Briggs Institute reviewer's manual. https://reviewersmanual.joannabriggs. org/2017. Accessed 12 Dec 2019.

30. Tricco AC, Lillie E, Zarin W, O'Brien KK, Colquhoun H, Levac D, et al. PRISMA Extension for Scoping Reviews (PRISMA-SCR): checklist and explanation. Ann Intern Med. 2018;169(7):467-73.

31. Bramer WM, Giustini D, de Jonge GB, Holland L, Bekhuis T. De-duplication of database search results for systematic reviews in EndNote. J Med Libr Assoc. 2016;104(3):240-3.

32. Glowacki K, Duncan MJ, Gainforth H, Faulkner G. Barriers and faciliators to physical activity and exercise among adults with depression: a scoping review. Ment Health Phys Act. 2017;13:108-19.

33. Ouzzani M, Hammady H, Fedorowicz Z, Elmagarmid A. Rayyan-a web and mobile app for systematic reviews. Syst Rev. 2016;5(1):210.

34. Istanboulian L, Rose L, Gorospe F, Yunusova Y, Dale CM. Barriers to and facilitators for the use of augmentative and alternative communication and voice restorative strategies for adults with an advanced airway in the intensive care unit: a scoping review. J Crit Care. 2020;57:168-76.

35. Weatherson KA, Gainforth HL, Jung ME. A theoretical analysis of the barriers and facilitators to the implementation of school-based physical activity policies in Canada: a mixed methods scoping review. Implement Sci. 2017;12(1):41.

36. Jahn P, Lakowa N, Landenberger M, Vordermark D, Stoll O. InterACTIV: an exploratory study of the use of a game console to promote physical activation of hospitalized adult patients with cancer. Oncol Nurs Forum. 2012;39(2):E84-90.

37. Haines KJ, Skinner EH, Berney S, Austin Health PSI. Association of postoperative pulmonary complications with delayed mobilisation following major abdominal surgery: an observational cohort study. Physiotherapy. 2013;99(2):119-25.

38. Kanaskie ML, Snyder C. Nurses and nursing assistants decision-making regarding use of safe patient handling and mobility technology: a qualitative study. Appl Nurs Res. 2018;39:141-7.

39. Jonsson LR, Ingelsrud LH, Tengberg LT, Bandholm T, Foss NB, Kristensen MT. Physical performance following acute high-risk abdominal surgery: a prospective cohort study. Can J Surg. 2017;60(6):12616

40. DeCuir-Gunby JT, Marshall PL, McCulloch AW. Developing and using a codebook for the analysis of interview data: an example from a professional development research project. Field Methods. 2011;23(2):136-55.

41. Cane J, O'Connor D, Michie S. Validation of the theoretical domains framework for use in behaviour change and implementation research. Implement Sci. 2012:7·37.

42. Granger CL, Parry SM, Denehy L, Remedios L. Evidence, education and multi-disciplinary integration are needed to embed exercise into lung cancer clinical care: a qualitative study involving physiotherapists. Physiother Theory Pract. 2018;34(11):852-60.

43. van Eijck SAH. Barriers and facilitators to implement function- focused care in hospitals: a mixed methods study: Faculty of Medicine Theses. Utrecht: Utrecht University; 2016.

44. Koenders N, van Oorsouw R, Seeger JPH, Nijhuis-van der Sanden MWG, van de Glind I, Hoogeboom TJ. "I'm not going to walk, just for the sake of walking...": a qualitative, phenomenological study on physical activity during hospital stay. Disabil Rehabil. 2020;42(1):78-85.

45. Alawadi ZM, Leal I, Phatak UR, Flores-Gonzalez JR, Holihan JL, Karanjawala $\mathrm{BE}$, et al. Facilitators and barriers of implementing enhanced recovery in colorectal surgery at a safety net hospital: a provider and patient perspective. Surgery. 2016;159(3):700-12.

46. Andreasen J, Soendergaard LN, Holst M. Factors affecting patient and nursing staff adherence to an integrated physical activity and nutritional 
intervention targeting functional decline on an acute medical ward: a qualitative study. Patient Prefer Adherence. 2018;12:1425-35.

47. Babine RL, Hyrkas KE, McKenzie CG, Wierman HR. Mobilizing older adults: a multi-site, exploratory and observational study on patients enrolled in the Hospital Elder Life Program (HELP). Geriatr Nurs. 2019;40(3):239-45.

48. Boltz M, Resnick B, Capezuti E, Shuluk J. Activity restriction vs. selfdirection: hospitalised older adults' response to fear of falling. Int J Older People Nurs. 2014;9(1):44-53.

49. Bradley G, Baker K, Bailey C. The meaning of rehabilitation: a qualitative study exploring perspectives of occupational therapists and physiotherapists working with older people in acute care. Disabil Rehabil. 2021;43(16):2295-303.

50. Chan E-Y, Hong LI, Tan Y-HG, Chua W-L. Older patients' participation in physical activity during hospitalization: a qualitative study of ward nurses' perceptions in an Asian context. Geriatr Nurs. 2019;40:91-8.

51. Clarke C, Stack C, Martin M. Lack of meaningful activity on acute physical hospital wards: older people's experiences. Br J Occup Ther. 2017;81:030802261773504.

52. Cook D, Tkaczyk A, Lutz K, McMullin J, Haynes RB, Douketis J. Thromboprophylaxis for hospitalized medical patients: a multicenter qualitative study. J Hosp Med. 2009;4(5):269-75.

53. Dahlke SA, Phinney A, Hall WA, Rodney P, Baumbusch J. Orchestrating care: nursing practice with hospitalised older adults. Int J Older People Nurs. 2015;10(4):252-62.

54. Dermody G, Kovach CR. Nurses' experience with and perception of barriers to promoting mobility in hospitalized older adults: a descriptive study. J Gerontol Nurs. 2017:43(11):22-9.

55. Eastham AJ, Cox D. Dementia-friendly wards: a review of the literature and pilot study of patient interaction and daily activity engagement. Int J Health Govern. 2017;22(1):25-36.

56. Gordge L, De Young J, Wiechula R. Reducing functional decline of older people in an acute-care setting: are we providing adequate care to maintain/optimise the functional status of our elder patients? Int J Evid Based Healthc. 2009;7(3):181-6.

57. Graf CL. Patient, Nurse, and Hospital Factors That Influence the Mobility of Hospitalized Older Adults. UCSF. ProQuest ID: Graf_ucsf_0034D_10751. REDACTED. Merritt ID: ark:/13030/m5xh1562. San Francisco; 2013. Retrieved from https://escholarship.org/uc/item/90z7x3tk.

58. Juneau A, Bolduc A, Nguyen P, Leclerc BS, Rousseau J, Dubé F, et al. Feasibility of implementing an exercise program in a geriatric assessment unit: the SPRINT program. Can Geriatr J. 2018;21(3):284-9.

59. King BJ, Steege LM, Winsor K, VanDenbergh S, Brown CJ. Getting patients walking: a pilot study of mobilizing older adult patients via a nurse-driven intervention. J Am Geriatr Soc. 2016;64(10):2088-94.

60. Kouchel D. Early mobility in hospitalized older adults: needs assessment, analysis and proposed intervention [Masters Thesis]. Carolina: University of North Carolina at Chapel Hill Graduate School; 2017.

61. Leak Bryant A, Walton AL, Pergolotti M, Phillips B, Bailey C, Mayer DK, et al. Perceived benefits and barriers to exercise for recently treated adults with acute leukemia. Oncol Nurs Forum. 2017;44(4):413-20.

62. Lim S, Ibrahim K, Dodds R, Purkis A, Baxter M, Rogers A, et al. Physical activity in hospitalised older people: the feasibility and acceptability of a volunteer-led mobility intervention in the SoMoVe ${ }^{\mathrm{TM}}$ study. Age Ageing. 2019;49(2):283-91.

63. Meesters J, Conijn D, Vermeulen HM, Vliet Vlieland T. Physical activity during hospitalization: activities and preferences of adults versus older adults. Physiother Theory Pract. 2019;35(10):975-85.

64. Moreno NA, de Aquino BG, Garcia IF, Tavares LS, Costa LF, Giacomassi IWS, et al. Physiotherapist advice to older inpatients about the importance of staying physically active during hospitalisation reduces sedentary time, increases daily steps and preserves mobility: a randomised trial. J Physiother. 2019;65(4):208-14.

65. Murphy JNF, Le Jeune I. Can an acute admission to hospital be an opportunity for healthcare professionals to provide physical activity advice? A qualitative study of patients' perspectives. Acute Med. 2018;17(1):10-7.

66. Nesbitt JC, Deppen S, Corcoran R, Cogdill S, Huckabay S, McKnight D, et al. Postoperative ambulation in thoracic surgery patients: standard versus modern ambulation methods. Nurs Crit Care. 2012;17(3):130-7.

67. Persson E, Anderberg P, Ekwall AK. A room of one's own--being cared for in a hospital with a single-bed room design. Scand J Caring Sci. 2015;29(2):340-6.
68. Rosbergen ICM, Brauer SG, Fitzhenry S, Grimley RS, Hayward KS. Qualitative investigation of the perceptions and experiences of nursing and allied health professionals involved in the implementation of an enriched environment in an Australian acute stroke unit. BMJ Open. 2017;7(12):e018226.

69. Sepulveda-Pacsi AL, Soderman M, Kertesz L. Nurses' perceptions of their knowledge and barriers to ambulating hospitalized patients in acute settings. Appl Nurs Res. 2016;32:117-21.

70. Tang CY, Taylor NF, Blackstock FC. Patients admitted with an acute exacerbation of chronic obstructive pulmonary disease had positive experiences exercising from the beginning of their hospital stay: a qualitative analysis. Chron Respir Dis. 2013;10(4):197-205.

71. Zisberg A, Agmon M, Gur-Yaish N, Rand D, Hayat Y, Gil E, et al. No one size fits all-the development of a theory-driven intervention to increase in-hospital mobility: the "WALK-FOR" study. BMC Geriatr. 2018;18(1):91.

72. Lim SH, Ang SY, Ong HK, Lee TZY, Lee TXL, Luo EZ, et al. Promotion of mobility among hospitalised older adults: an exploratory study on perceptions of patients, carers and nurses. Geriatr Nurs. 2020;41(5):608-14.

73. Lee-Steere K, Liddle J, Mudge A, Bennett S, McRae P, Barrimore SE. "You've got to keep moving, keep going": understanding older patients' experiences and perceptions of delirium and nonpharmacological delirium prevention strategies in the acute hospital setting. J Clin Nurs. 2020;29(13-14):2363-77.

74. Rasmussen RL, Holst M, Nielsen L, Villumsen M, Andreasen J. The perspectives of health professionals in Denmark on physical exercise and nutritional interventions for acutely admitted frail older people during and after hospitalisation. Health Soc Care Community. 2020;28(6):2140-9.

75. Kalisch BJ. Missed nursing care: a qualitative study. J Nurs Care Qual. 2006;21(4):306-13 quiz 14-5.

76. Doherty-King B, Bowers BJ. Attributing the responsibility for ambulating patients: a qualitative study. Int J Nurs Stud. 2013;50(9):1240-6.

77. Douglas CH, Douglas MR. Patient-centred improvements in health-care built environments: perspectives and design indicators. Health Expect. 2005;8(3):264-76

78. Douglas $\mathrm{CH}$, Douglas MR. Patient-friendly hospital environments: exploring the patients' perspective. Health Expect. 2004;7(1):61-73.

79. Lafreniere S, Folch N, Dubois S, Bedard L, Ducharme F. Strategies used by older patients to prevent functional decline during hospitalization. Clin Nurs Res. 2017;26(1):6-26.

80. Lyon A, Solomon MJ, Harrison JD. A qualitative study assessing the barriers to implementation of enhanced recovery after surgery. World I Surg. 2014:38(6):1374-80

81. Holst M, Hansen LA, Pedersen LA, Paulsen S, Valentinsen CD, Kohler M. Physical activity in hospitalized old medical patients; how active are they, and what motivates to physical activity. J Aging Res Clin Pract. 2015;4(2):116-23.

82. Gillis A, MacDonald B, Maclsaac A. Nurses' knowledge, attitudes, and confidence regarding preventing and treating deconditioning in older adults. J Contin Educ Nurs. 2008;39(12):547-54.

83. Cheah S, Presnell S. Older people's experiences of acute hospitalisation: an investigation of how occupations are affected. Aust Occup Ther J. 2011;58(2):120-8

84. Parke B, Chappell NL. Transactions between older people and the hospital environment: a social ecological analysis. J Aging Stud. 2010;24(2):115-24.

85. van der Leeden M, Huijsmans R, Geleijn E, de Lange-de Klerk ES, Dekker J, Bonjer $\mathrm{HJ}$, et al. Early enforced mobilisation following surgery for gastrointestinal cancer: feasibility and outcomes. Physiotherapy. 2016;102(1):103-10.

86. van Delft L, Bor P, Valkenet K, Slooter A, Veenhof C. The effectiveness of hospital in motion, a multidimensional implementation project to improve patients' movement behavior during hospitalization. Phys Ther. 2020;100(12):2090-8.

87. van Dijk-Huisman HC, Weemaes ATR, Boymans T, Lenssen AF, de Bie RA. Smartphone App with an accelerometer enhances patients' physical activity following elective orthopedic surgery: a pilot study. Sensors (Basel). 2020;20(15):4317.

88. Koenders N, Potkamp-Kloppers S, Geurts Y, Akkermans R, Nijhuis-van der Sanden MWG, Hoogeboom TJ. Ban Bedcentricity: A Multifaceted Innovation to Reduce Sedentary Behavior of Patients During the Hospital Stay. Phys Ther. 2021;101(7). 
89. Klein LM, Young D, Feng D, et al. Increasing patient mobility through an individualized goal-centered hospital mobility program: a quasi-experimental quality improvement project. Nurs Outlook. 2018;66:254-62.

90. Probasco JC, Lavezza A, Cassell A, Shakes T, Feurer A, Russell H, et al. Choosing wisely together: physical and occupational therapy consultation for acute neurology inpatients. Neurohospitalist. 2018;8(2):53-9.

91. Cohen Y, Zisberg A, Chayat Y, Gur-Yaish N, Gil E, Levin C, et al. Walking for better outcomes and recovery: the effect of walk-for in preventing hospital-associated functional decline among older adults. J Gerontol A Biol Sci Med Sci. 2019;74(10):1664-70.

92. Garne-Dalgaard A, Mann S, Bredahl TVG, Stochkendahl MJ. Implementation strategies, and barriers and facilitators for implementation of physica activity at work: a scoping review. Chiropr Man Therap. 2019;27:48.
93. Huijg JM. Towards the effective introduction of physical activity interventions in primary health care. Enschede: University of Leiden; 2014.

94. Little EA, Presseau J, Eccles MP. Understanding effects in reviews of implementation interventions using the Theoretical Domains Framework. Implement Sci. 2015;10:90.

95. Prior M, Guerin M, Grimmer-Somers K. The effectiveness of clinical guideline implementation strategies--a synthesis of systematic review findings. J Eval Clin Pract. 2008;14(5):888-97.

\section{Publisher's Note}

Springer Nature remains neutral with regard to jurisdictional claims in published maps and institutional affiliations.
Ready to submit your research? Choose BMC and benefit from:

- fast, convenient online submission

- thorough peer review by experienced researchers in your field

- rapid publication on acceptance

- support for research data, including large and complex data types

- gold Open Access which fosters wider collaboration and increased citations

- maximum visibility for your research: over $100 \mathrm{M}$ website views per year

At BMC, research is always in progress.

Learn more biomedcentral.com/submissions 